\title{
Challenges and Opportunities toward the Decade of Healthy Ageing in the Post-pandemic Era
}

\author{
Jae-Young Lim \\ Department of Rehabilitation Medicine, Seoul National University Bundang Hospital, Seoul National University College of Medicine, Seongnam, Korea
}

An 84-year-old woman with gait disturbance attributable to frailty and sarcopenia was admitted for rehabilitation. She exhibited functional improvement and continued outpatient rehabilitation in mid-February 2020. At that time, coronavirus disease 2019 (COVID-19) cases rapidly increased and strict social distancing was imposed country-wide. She could not visit our outpatient center for fear of infection and was lost to follow-up for over 3 months. Consequently, her mobility, which had been steadily improving, suddenly deteriorated. In general, older people are the most vulnerable victims of the COVID-19 pandemic, having not only virus-caused death and severe morbidity but also overall functional declines owing to their isolation and lack of care. In many countries, long-term care facilities are the epicenters of COVID-19 outbreaks. In such constant emergencies, it is nearly impossible to deliver interventions for geriatric conditions in clinical settings. ${ }^{1)}$ During the pandemic, "nonessential" community services were suspended, including senior activity and dementia daycare centers, geriatric day hospitals, and outreach rehabilitation programs. ${ }^{2)}$ Declines in physical activity and exercise have been reported in almost $50 \%$ of older adults living independently. ${ }^{3)}$ Soon, we expect to see studies on the extent of physical deterioration in older adults during the pandemic. The year-long failure of treatment for older patients who require timely care will also soon negatively impact population health indicators.

The United Nations declared 2020-2030 as the Decade of Healthy Ageing; associated activities aimed at ensuring health at all ages and involving a range of co-operating professionals are required to meet this goal. ${ }^{4)}$ A decade of concerted global action is urgently needed to ensure that older adults enjoy dignity, equality, and a healthy environment. However, one year into this project, the pandemic has overshadowed the healthy aging agenda. Indeed, the pandemic has highlighted the yawning gaps in policies, systems, and services. While some countries will emerge from the pandemic late in 2021, other countries will not.

How can we overcome the crisis in healthy aging during the pandemic? The need to insulate older adults from COVID-19 and optimize their overall health must be balanced. The Asian Working Group for Sarcopenia has made relevant recommendations. ${ }^{2)}$ The American Geriatric Society Healthy Aging Special Interest Group has also provided guidance to geriatric healthcare professionals; the suggested strategies would enhance healthy aging even in the era of COVID-19. ${ }^{5)}$ Despite the World Health Organization's concerns about the effects of the pandemic on the health of older people, we must renew the Decade of Healthy Ageing project. $\left.{ }^{6}\right)$ The pandemic will continue to present serious challenges worldwide for many years. However, the crisis has forced governments, businesses, and societies to change rapidly, and some of these changes are positive. Many participants noted that the pandemic afforded an opportunity to re-set the current long-term care model, thus rendering it more person centered, with a focus on healthy aging. Furthermore, we have a unique opportunity to advocate for policies that invest in a long-term care model that views older people as vital contributors to healthy communities rather than simply the cost that society must bear. The provision of person-centered, non-discriminatory, accessible, integrated primary health and social care should be increased. Communities should be supported in terms of developing or maintaining the capacities of older people, both during the pandemic and beyond. Scale up of integrated care for older people will also require investment in and reinforcement of health systems. ${ }^{7)}$

Alternative models of care are urgently needed during the pandemic. Telephone or video visits are also valuable. An information and communication technology-based integrated service model has been developed to provide effective management, enable consultation with distant professionals, and share medical information between acute care hospitals and long-term care institutions. ${ }^{8)}$ The 
Korean Ministry of Health and Welfare temporarily allowed telephone-based consultation and prescription at the beginning of the COVID-19 outbreak. Community- or home-based medical care, including patient education, discharge care planning, and homebased monitoring, has also become crucial. ${ }^{9)}$ Essential medical care should continue during strict social distancing and when older adults cannot visit healthcare facilities. Telemedicine and telerehabilitation are developing rapidly as alternative models of care. ${ }^{2)}$ Geriatric care professionals assist patients and families to optimize health by referring them to digital resources whenever possible. While such resources were available before the pandemic, almost all have been extensively improved because it has become essential to interact in a virtual world. ${ }^{5)}$ More personalized and inclusive services will more effectively meet the needs of older adults, reduce the demand for hospital beds, and lower the risks of morbidity and mortality. Thus, clinical evidence and healthcare systems supporting such types of services should be developed via well-designed high-quality studies.

Annals of Geriatric Medicine and Research welcomes innovative research results of the professional geriatric community on healthy aging in the post-pandemic era, which will stimulate informative discussions. Future studies should continue to explore unprecedented challenges imposed by the pandemic and develop new interventions against another global pandemic impacting the health and social functioning of older adults. As we have entered the Decade of Healthy Ageing, governments, businesses, and community organizations worldwide must rise to the challenge. Everyone should be able to enjoy healthier, happier, and longer lives.

\section{ACKNOWLEDGMENTS}

\section{CONFLICT OF INTEREST}

The author claims no conflicts of interest.

\section{REFERENCES}

1. Cesari M, Proietti M. Editorial: Geriatric medicine in Italy in the time of COVID-19. J Nutr Health Aging 2020;24:459-60.
2. Lim WS, Liang CK, Assantachai P, Auyeung TW, Kang L, Lee WJ, et al. COVID-19 and older people in Asia: Asian Working Group for Sarcopenia calls to actions. Geriatr Gerontol Int 2020; 20:547-58.

3. Visser M, Schaap LA, Wijnhoven HA. Self-reported impact of the COVID-19 pandemic on nutrition and physical activity behaviour in Dutch older adults living independently. Nutrients 2020;12:3708.

4. Lloyd-Sherlock P, Kalache A, Kirkwood T, McKee M, Prince M. WHO's proposal for a decade of healthy ageing. Lancet 2019; 394:2152-3.

5. Batsis JA, Daniel K, Eckstrom E, Goldlist K, Kusz H, Lane D, et al. Promoting healthy aging during COVID-19. J Am Geriatr Soc 2021;69:572-80.

6. Kurjak A, Jakovljevic M, Masic I, Stanojevic M. Healthy aging in the time of corona pandemic. Med Arch 2021;75:4-10.

7. Charles AE, Ewbank L. The road to renewal: five priorities for health and care. [Internet]. London, UK: The King's Fund; 2021 [cited 2021 Jun 24]. Available from: https://www.kingsfund.org.uk/publications/covid-19-road-renewal-health-andcare.

8. Choi JY, Kim KI, Lim JY, Ko JY, Yoo S, Kim H, et al. Development of health-RESPECT: an integrated service model for older long-term care hospital/nursing home patients using information and communication technology. Ann Geriatr Med Res 2020;24:27-34.

9. Jang SN, Kim CO. Care inequality among older adults during the COVID-19 pandemic. Ann Geriatr Med Res 2020;24:22931.

Corresponding Author: Jae-Young Lim, MD, $\mathrm{PhD}$

Department of Rehabilitation, Seoul National University Bundang Hospital, Seoul National University College of Medicine, 82 Gumi-ro, 173beon-gil, Bundang-gu, Seongnam 13620, Korea

E-mail: drlim1@snu.ac.kr

ORCID: https://orcid.org/0000-0002-2906-3094

Received: June 17, 2021; Accepted: June 18, 2021 